$\underset{\text { NATIONAL LABORATORY }}{\text { BROOKHYEN }}$

BNL-73462-2005-CP

\title{
Global Decoupling on the RHIC Ramp
}

\author{
Y. Luo, P. Cameron, A. DellaPenna, W. Fischer, J. Laster, A. \\ Marusic, F. Pilat, T. Roser, D. Trbojevic
}
Presenfed af the Particle Accelerator Conference(PAC'05) Knoxville, Tennessee
May 16-20, 2005

\author{
Collider-Accelerator Department \\ Brookhaven National Laboratory \\ P.O. Box 5000 \\ Upton, NY I1973-5000 \\ www.bnt.gov \\ Managedby \\ Brookhaven Science Associates, LLC \\ for the United States Department of Energy under \\ Contract No. DE-AC02-98CH10886
}

This is a preprint of a paper intended for publication in a journal or proceedings. Since changes may be made before publication, this preprint is made availablewith the understanding that it will not be cited or reproduced without the permission of the author. 


\section{DISCLAIMER}

This report was prepared as an account of work sponsored by an agency of the United States Government. Neither the United States Government nor any agency thereof, nor any of their employees, nor any of their contractors, subcontractors, or their employees, makes any warranty, express or implied, or assumes any legal liability or responsibility for the accuracy, completeness, or any third party's use or the results of such use of any information, apparatus, product, or process disclosed, or represents that its use would not infringe privately owned rights. Reference herein to any specific commercial product, process, or service by trade name, trademark, manufacturer, or otherwise, does not necessarily constitute or imply its endorsement, recommendation, or favoring by the United States Government or any agency thereof or its contractors or subcontractors. The views and opinions of authors expressed herein do not necessarily state or reflect those of the United States Government or any agency thereof.

FOR UNCLASSIFIED, UNLIMITED STIPRODUCTS

Available electronically at:

OSTI:

http://www.os ti.gov/bridge

Available for a processing fee to U.S. Department of Energy and its contractors, in paper from:

U.S. Department of Energy

Office of Scientific and Technical Information

P.O. Box 62

Oak Ridge, TN 37831

Phone: (865) 576-8401

Facsimile: (865) 576-5728

E-mail: reports@adonis.osti.gov

National Technical Information Service (NTIS):

Available for sale to the public from:

U.S. Department of Commerce

National Technical Information Service

5285 Port Royal Road

Springfield, VA 22131

Phone: (800) 553-6847

Facsimile: (703) 605-6900

Online ordering: http://www.ntis.gov/ordering.htm

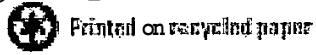




\title{
Global Decoupling on the RHIC Ramp*
}

\author{
Y. Luo, P. Cameron, A. DellaPenna, W. Fischer, J. Laster, A. Marusic, F. Pilat, T. Roser, D. Trbojevic \\ Brookhaven National Laboratory, Upton, NY 11973, USA
}

\begin{abstract}
The global betatron decoupling on the ramp is an important issue for the operation of the Relativistic Heavy Ion Collider (RHIC), especially in the RHIC polarized proton (pp) run. To avoid the major betatron and spin resonances on the ramp, the betatron tunes are constrained. And the rms value of the vertical closed orbit shouldbe smallerthan $0.5 \mathrm{~mm}$. Both require the global coupling on the ramp to be well corrected. Several ramp decoupling schemes were found and tested at RHIC, like N-turn map decoupling, three-ramp correction, coupling amplitudemodulation, and coupling phase modulation. In this article, the principles of these methods are shortlyreviewed and compared. Among them, coupling angle modulation is a robust and fast one. It has been applied to the global decoupling in the routine RHIC operation.
\end{abstract}

\section{INTRODUCTION}

The betatron coupling on the ramp can be identified with the turn-by-turnbeam position monitor (BPM) data from the tune meter kicked beam. The tune meter kicker kicks one bunch on the ramp every 2 second. Under the coupled situation, the transverseoscillation amplitudebeating of the horizontal and vertical betatron motions are observed. And normally there are doublepeaks in the fastFourier transformation (FFT) spectrum of the horizontal and vertical betatron oscillations.

The global decoupling on the ramp is more difficultthan that at injection and store. Besides the non-stop energy acceleration, the beam optics evolves. The snapback, transition, and beta squeezing all pose challenges to the global decoupling. The movement of the closed orbit, especially the vertical closed orbit in the sextupoles, changes the coupling on the ramp. The RHIC pp run's main ramp takes about 220 seconds. And the rotator ramp at the energy flattop takes about 430 seconds. Therefore, a fast and robust global decouplingschemeand reliableinstrumentationsare needed.

The conventional skew quadrupole strength scan is not applicable for the ramp coupling correction. As a logical extension, the skew quadrupole modulation was put forth to fulfill the global decoupling on the ramp. Either the coupling amplitude or coupling angle can be modulated [ 1 , $\mathbf{2 , 3 ]}$. The coupling amplitude modulation gives the residval coupling'sprojections onto the skew quadrupole modulation directions. The coupling angle modulation gives the coupling correction strengths. The tune changes during the skew quadrupole modulation are tracked with the

*Work supported by U.S. DOE under contract No DE-ACO298CH10886 phase lock loop(PLL) tunemeasurementsystem [4,5]. Besides the skew quadrupolemodulation, $\mathrm{N}$-turn transfermap decoupling and three-ramp decoupling schemes were also tested at the RHIC. All the above schemes are used in the feed-forwardmode. The global decoupling in the feedback mode is being investigatedat RHIC based on the six global coupling observables. The phase loop and amplituderatio loop are promising for the future decoupling feedback [6].

In the following, we first quickly review the principles for the decouplingmethods on the ramp. Then, decoupling exampleswith the coupling angle modulation are given.

\section{RAMP DECOUPLING SCHEMES}

\section{$N$-turn transfer maps [7]}

At one point in the ring, the $4 \times 4$ linear transfer matrix of the betatron motion is given by

$$
\mathbf{M}=\left(\begin{array}{ll}
\mathbf{A} & \mathbf{B} \\
\mathbf{C} & \mathbf{D}
\end{array}\right)
$$

To decouple globally is equivalent to meet $\boldsymbol{C}+\overline{\mathbf{B}}=\boldsymbol{O}$. To be able to observe the maximum effect of the transverse beating, Fischer calculates the N-turn transfer matrix $\mathbf{M}^{N}$ instead of the one-turn transfer matrix M. $N$ is the revolution turn number given by the half transverse beating period due to the coupling,

$$
N \sim \frac{\pi}{\left|Q_{1}-Q_{2}\right|} .
$$

Here $Q_{1,2}$ are the two eigentunes. $(\boldsymbol{C}+\overline{\mathbf{B}})$ can be determined from the $\mathrm{N}$-turn matrix $\mathrm{M}^{N}$,

$$
\mathbf{C}+\overline{\mathbf{B}}=\left(\mathbf{C}_{N}+\overline{\mathbf{B}}_{N}\right) \frac{\cos Q_{1}-\cos Q_{2}}{\cos \left(N Q_{1}\right)-\cos \left(N Q_{2}\right)}
$$

The two eigentunes are obtained from the fast Fourier transformation of the turn-by-turn BPM data. The N-turn transfer matrix $\mathbf{M}^{N}$ is obtained through the fitting. Knowing $\boldsymbol{C}+\overline{\mathbf{B}}$, the one-turn matrix is decoupled with the skew quadrupole families according to the thin skew quadrupole and weak coupling approximations.

This method has been successfully applied at injection, where it can decouple within seconds after a bunch was taken. However, so far it failed to work on ramps. In previous years the quality of the turn-by-turn orbit data did not allow a reliable fit of the $\mathrm{N}$-turn map. These difficulties were resolved, and the same $\mathrm{N}$-turn map is now calculated for consecutive ramps. The method calculates the effect of the skew correctors from an online model. At higher energies (and lower $\beta^{*}$ ), the model is not accurate enough any 
more, and the wrong corrector strenght are calculated. To overcome this problem, the phases of the skew correctors may need to be fitted from experimental data.

\section{3-ramp correction}

From the linear difference coupling's Hamiltonian perturbation theory $[8,9,10,11]$, the eigentune split AQ is

$$
|\Delta Q|=\sqrt{\Delta^{2}+\left|C^{-}\right|^{2}} .
$$

Here $\mathrm{A}$ is the uncoupled tune split. $\mathrm{C}^{-}$is the coupling coefficient,

$$
C^{-}=\left|C^{-}\right| e^{\imath \chi}=\frac{1}{2 n} \oint \sqrt{\beta_{x} \beta_{y}} k_{s} e^{i\left(\Phi_{x}-\Phi_{y}-\Delta \frac{2 \pi s}{L}\right)} d l .
$$

$\left|C^{-}\right|$is the coupling amplitude, $\chi$ is the angle of the coupling. $C^{-}$normally is a complex number.

The total coupling coefficient $C_{t o t}^{-}$in the ring is

$$
C_{\text {tot }}^{-}=C_{\text {res }}^{-}+C_{\text {int }}^{-} \text {, }
$$

where $C_{\text {res }}^{-}$is the residual coupling, $C_{\text {int }}^{-}$is the introduced coupling coefficient.

In Eq. (4), there are three real unknowns, A, real and imaginary parts of $C^{-}$. Therefore, Trbojevic suggestedusing three ramps to determine the residual coupling at one specific ramp time point. For each ramp, we change the settings of the skew quadruople families, the introduced $C_{i n t}^{-}$ can be calculated from the optics model.

The shortcoming of this method is apparent. It requires three ramps. And this method has tight connections to the optics since $C_{i n t}^{-}$is calculated from the optics model. This method is the last choice for the ramp coupling correction.

\section{Coupling amplitude modulation [2]}

Skew quadrupolemodulation was proposed for the ramp coupling correction. The skew quadrupolemodulation can be either coupling amplitudemodulation or coupling angle modulation. Coupling angle modulation witl be discussed in next section in detailed.

The introduced coupling by the coupling amplitude modulation is

$$
C_{\text {mod }}^{-}=C_{m o d, a m p}^{-} \sin (2 \pi f t) .
$$

$f$ is the modulationfrequency. $C_{m o d, a m p}^{-}$is the modulation amplitude.

Then, accordingto Eq. (4), the eigentune split during the modulation is

$$
\begin{aligned}
\left(Q_{1}-Q_{2}\right)^{2}= & \Delta^{2}+\left|C_{\text {res }}^{-}\right|^{2}+\frac{1}{2}\left|C_{m o d, a m p}^{-}\right|^{2} \\
& +2\left|C_{\text {res }}^{-}\right|\left|C_{\text {mod,amp }}^{-}\right| \cos (\varphi) \sin (2 \pi f t) \\
& -\frac{1}{2}\left|C_{\text {mod, }, a m p}^{-}\right|^{2} \cos (4 \pi f t),
\end{aligned}
$$

where $\varphi$ is the angle difference between $C_{\text {res }}^{-}$and $C_{\text {mod,amp }}^{-}\left|C_{\text {res }}^{-}\right| \cos (\varphi)$ is the projection of the residual coupling onto the modulation coupling direction.

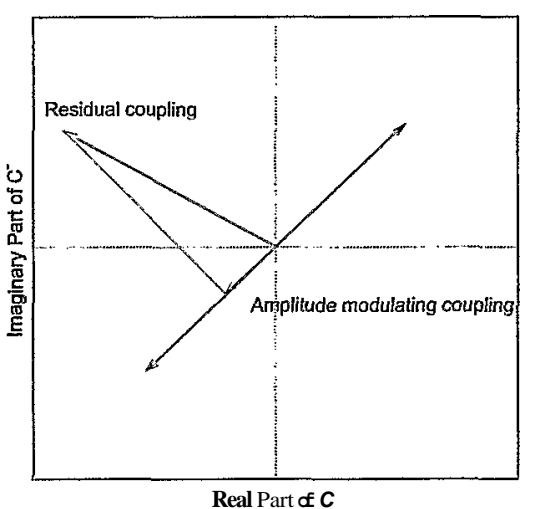

Figure 1: Schematic plot of coupling amplitude modulation.

We define the projection ratio $k$ as

$$
\kappa=\frac{\left|C_{\text {res }}^{-}\right| \cos (\varphi)}{\left|C_{\text {mod,amp }}^{-}\right|}
$$

According to Eq. (8), it can be determinedfrom the FFT of $\left(Q_{1}-Q_{2}\right)^{2}$. If the $1 f$ and $2 f$ peaks' amplitudes are $A_{1 f}$ and $A_{2 f}$, respectively,

$$
|\kappa|=\frac{A_{1 f}}{4 A_{2 f}} .
$$

Knowing the projections of the residual coupling onto at least two skew quadrupolemodulation direction, the residual coupling coefficient can be determined and eventually compensated. The schematic plot of coupling amplitude modulation is shown in Figure. 1.

This method was tested in RHIC in 2004. The skew quadrupole modulation frequency for the ramp was chosen as $0.2 \mathrm{~Hz}$. Linear regression fitting was used to greatly reduce the modulationtime to below 10 seconds. However, it is still not fast and robust enough for the ramp coupling correction.

\section{COUPLING ANGLE MODULATION}

Coupling angle modulation was proved to be a fast and robust global decoupling method. It was experimentally verified at RHIC injection and store, and has also been applied to the RHIC ramp.

\section{Principle [1]}

This methodmodulatestwo orthogonalskew quadrupole families. The coupling coefficients contributed by the orthogonal families differ by $90^{\prime \prime}$. If their coupling coefficient modulation amplitudes and modulation frequencies are same, and there is a $90^{\circ}$ differencein their initial modulation phases, the total introduced coupling coefficient is

$$
C_{m o d}^{-}=\left|C_{m o d, a m p}^{-}\right| \cdot e^{i 2 \pi f t_{7}}
$$




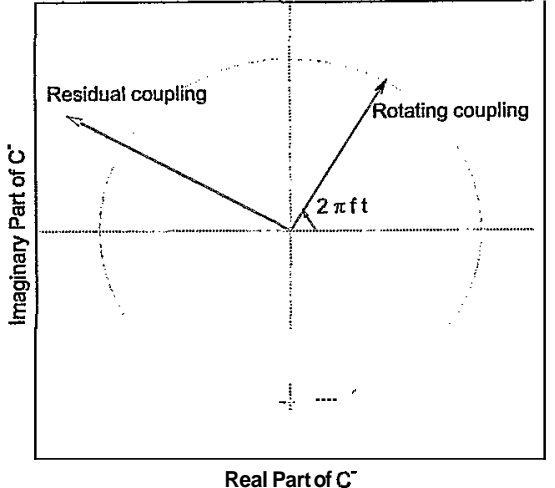

Figure 2 Schematicplot of the coupling angle modulation.

where $f$ is the modulation frequency, and $\left|C_{m o d, a m p}^{-}\right|$is the coupling modulation amplitude. Figure. 2 shows the schematic plot of the coupling angle modulation.

According to Eq. (4), the tune split's square during the modulation is

$$
\begin{aligned}
|\Delta Q|^{2}= & \Delta^{2}+\left|C_{\text {res }}^{-}\right|^{2}+\left|C_{\text {mod,amp }}^{-}\right|^{2} \\
& +2\left|C_{\text {res }}^{-}\right|\left|C_{\text {mod,amp }}^{-}\right| \cos \left(2 \pi f t-\phi_{\text {res }}\right) .
\end{aligned}
$$

Assuming the rotating coupling's amplitude $\left|C_{\text {mod,amp }}^{-}\right|$ is constant duringthe coupling anglemodulation, we define

$$
\left|C_{\text {res,amp }}^{-}\right|=k\left|C_{m o d, a m p}^{-}\right| .
$$

$h$ is a non-negativenumber. Then, the maximum and minimum tune split's squares are

$$
\begin{aligned}
& \Delta Q_{\text {min }}^{2}=\Delta^{2}+(k-1)^{2} \cdot\left|C_{\text {mod,amp }}^{-}\right|^{2}, \\
& \Delta Q_{\text {max }}^{2}=\Delta^{2}+(k+1)^{2} \cdot\left|C_{\text {mod,amp }}^{-}\right|^{2} .
\end{aligned}
$$

Together with the tune split square $\Delta Q_{0}^{2}$ without modulation,

$$
\Delta Q_{0}^{2}=\Delta^{2}+k^{2} \cdot\left|C_{m o d, a m p}^{-}\right|^{2},
$$

the factor $k$ is determined,

$$
k=\left[4\left(\frac{\Delta Q_{\max }^{2}-\Delta Q_{0}^{2}}{\Delta Q_{\max }^{2}-\Delta Q_{\min }^{2}}-\frac{1}{2}\right)\right]^{-1} .
$$

The factor $k$ has a significant role in determining of the correction strengths of the global coupling. The minimum tune split is obtained when the rotating coupling takes the opposite directionto the residual coupling. Therefore, the right decoupling skew quadrupolestrengths' combination is given at the minimum tune split time stamp. Since the skew quadrupolemodulation currents are known at that time point, according to Eq. (13) and Eq. (17) , the global decoupling strengths are determined. That is, The correction strengths are the modulating skew quadrupole strengths at the minimum tune split multiplied by the factor $k$.

This method links the global coupling correction strengths with the modulating skew quadruple family strengths through the rotating coupling. The ratios of the modulating skew quadrupolefamilies give the right decoupling direction. This methodjust needs the minimum and maximum tune split during the modulation, so it is insensitive to the detailed PLL tunes. And the method is also robust against model errors. The exact modulation amplitudes and modulation phases for the skew quadrupolemodulation are not required. Since the minimum andmaximum tune splits can be obtained in one modulation period, this method is fast, too.

\section{RHIC Modulation Setup}

Each RHIC ring has three correction skew quadrupole families, F1, F2 and F3, each having 12 skew quadrupole magnets, with four activating power supplies. To produce the rotating coupling coefficient, we use skew quadrupole familiesF1 and F3 to generate a new skew quadrupole family (F1F3) that is orthogonal to the coupling contribution only from family F2. To equalize families(F1F3) and F2's coupling modulation amplitudes, the amplitudes of modulation strengths for families F1 and F3 are set to $\frac{1}{\sqrt{3}}$ of that of family F2. To get a $90^{\circ}$ difference in the initial modulation phases, family $\mathrm{F} 2$ modulation starts a quarter period earlier than families F1 and F3.

\section{Verifying at injection and store}

Coupling angle modulation correction was first tested at RHIC injection and store. Here we gave an example of decoupling in the RHIC Blue ring at store. Fig. 3 shows the PLL tune during one complete coupling angle modulation coupling correction. After correction, the eigentune split was greatly reduced. FIG. 4 shows the modulating skew quadrupolepower supply currents and the tune split square $|\Delta Q|^{2}$. The maximum and minimum tune splits were searched between the two vertical grid lines. Before the coupling correction, $\Delta Q_{\min }=0.0078$. After coupling correction, $\Delta Q_{\min }=0.0006$.

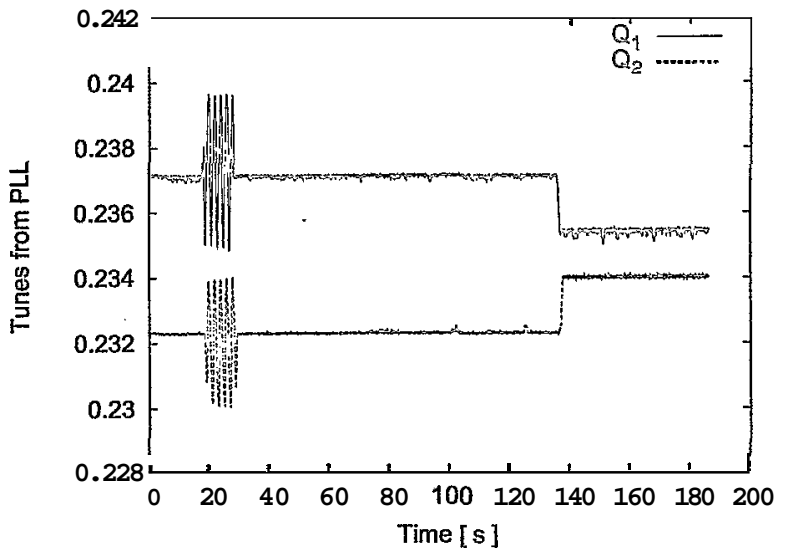

Figure 3: The tunes during the coupling angle modulation at Blue store. 


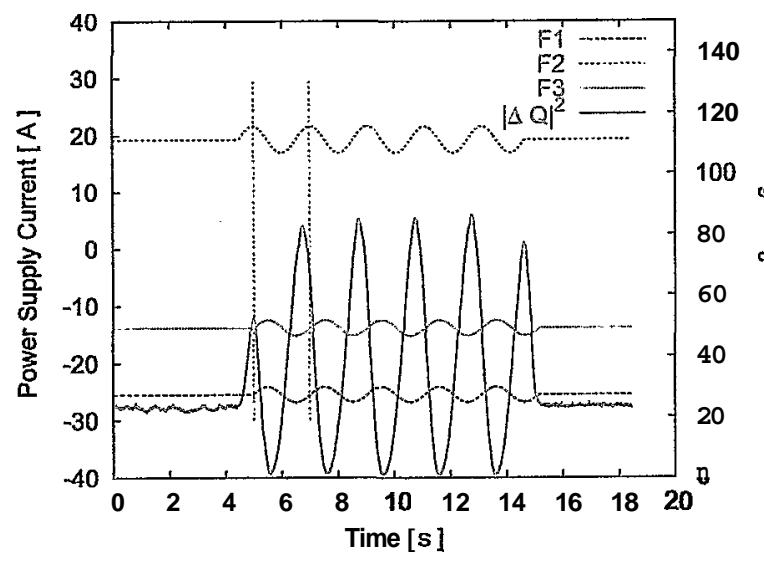

Figure 4: The coupling correction strength searching.

\section{Applying to ramp}

The whole RHIC 2005 pp run's main ramp takes about 220 seconds. The rotator ramp takes about 430 seconds at energy flattop. Fig. 5 shows the yellow tunes from the coupling angle modulation in the first ramp fill 6817. In Fig. 5, only the tunes in the first 140 seconds of the energy ramp are shown. After 140 secondsfrom the ramp starting point, there is a tune swing for both tunes. The skew quadrupole modulationfrequencyis $0.2 \mathrm{~Hz}$. In Fig. 5, each modulation took 15 seconds, or three modulationperiods.

Fig. 6 shows the second ramp tune data with coupling corrections. The correction strengths from the first ramp were put into this ramp fill 6818. Comparingthe tune splits before and after coupling correction, the coupling are reduced. After the global decoupling, the tunes were easy to move in the ramp development.

During the application of the coupling angle modulation correction to the ramp, we found sometime it is hard to get the exact $k$ factor for the full coupling correction like at injection and store. This is because of the tune changes. According to the experiences at RHIC, the most important issue for the coupling angle modulation correction on the ramp is to obtain valid PLL tune data. PLL tune losinglock was seen under some coupling situation.

\section{CONCLUSION}

The global betatron decoupling on the ramp is an important issue for the operation of the Relativistic Heavy Ion Collider $(\boldsymbol{R H I C})$, especially in the RHIC polarized proton nun. Several decoupling schemes were found and tested at RHIC, like N-turnmap decoupling, three-ramp correction, coupling amplitude modulation, and coupling phase modulation. Among them, coupling angle modulation is a robust and fast, and the most promising of the tried methods. It has been applied to the RHIC'05 pp run. The possible global decoupling in the feedback mode with eigen mode amplitude ratios and phase differences are being tested at the RHIC.

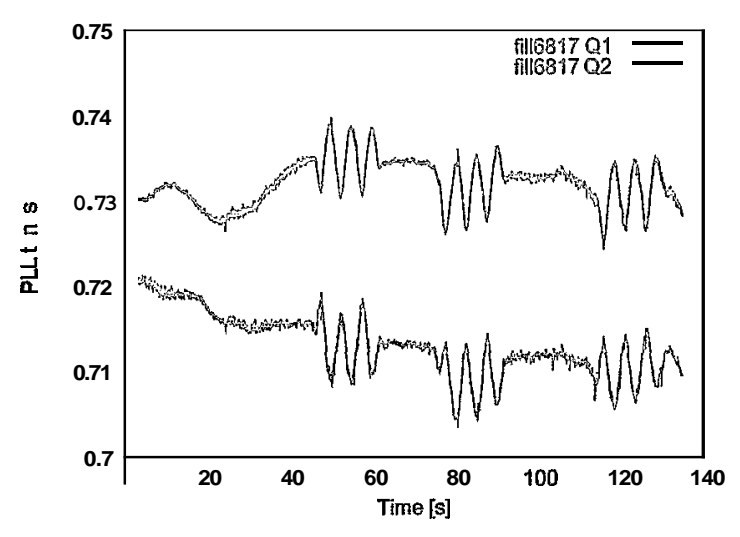

Figure 5: The tunes from the coupling angle modulation.

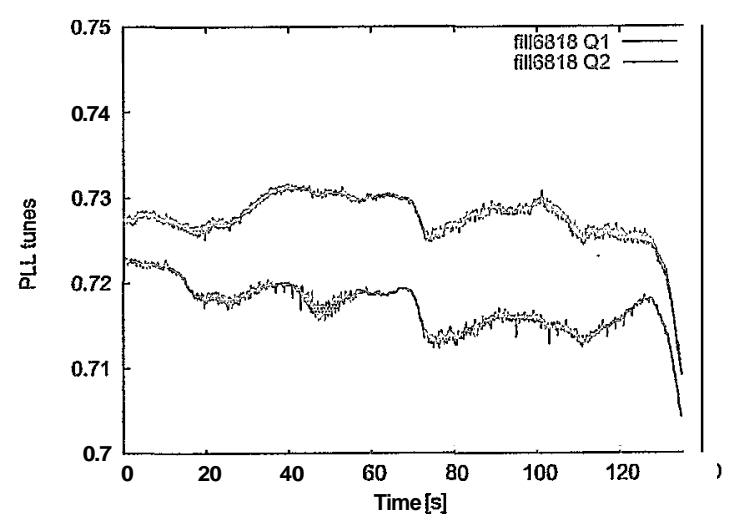

Figure 6 The tunes after the coupling corrections.

\section{REFERENCES}

[1] Y. Luo, F. Pilat, D. Trbojevic, T. Roser, J. Wei, Principle of Global Decoupling on the Ramp, BNL C-AD AP Note 165, Sept.,2004

[2] Y. Luo, et al., Phys. Rev. ST Accel. Beams 8,014001 (2005).

[3] Y. Luo, et al., robust andfastglobaldecouplingwithcoupling angle modulatio, submitted to Phys. Rev. ST Accel. Beams.

[4] P. Cameron, J. Cupolo, et al., 'RHIC Third GenerationPLL Tune System", inProceedings of the 2004 European Particle Accelerator Conference, Lucerne, Switzerland, p524.

[5] P. Cameron, P. Cerniglia, et al., "Tune Feedback at RHIC", in Proceedings of the 2004 European Particle Accelerator Conference, Lucerne, Switzerland, p1294.

[6] Y. Luo, P. Cameron, S.Peggs, D. Trbojevic, Possible phase loopfor the GlobalDecoupling on the Ramp, BNL $C$-AD AP Note 174, Sept., 2004

[7] W. Fischer, Phys. Rev. ST Accel. Beams 554001 (2002).

[8] S. Scoch, CERN Report No. 57-20, 1957 (unpublished).

[9] G. Guignard, CERN Report No. 76-06,1976 (unpublished).

[10] G. Guignard, Phys. Rev. E51, p6104, 1995.

[11] H. Wiedemann, Particle Accelerator Physics $I$, Nonlinear and Higher-OrderBeam Dynamics, Springer-Verlag, 1995. 